Pensamiento Crítico Vol. 19 № 1, pp. 33-53

\title{
Un modelo de predicción de crisis financieras en los mercados emergentes: 1970 - 2009
}

A prediction model of financial crises in emerging markets: 1970 - 2009

Alfonso Leonel Ayala Loro*

\section{Resumen}

En el presente documento se describe uno de los modelos de predicción de crisis financieras más importantes para mercados emergentes: el modelo de señales, asimismo se muestran los resultados de la aplicación de este modelo, usando datos mensuales desde 1970 hasta el primer trimestre del 2009. El modelo propuesto está basado en el enfoque de Kaminsky, Lizondo y Reinhart (1998). Se obtiene una identificación de los principales factores determinantes de crisis financieras (en el sentido empírico que se utiliza en el presente trabajo), entendida como una aproximación a la probabilidad de crisis en el corto plazo.

Número de Clasificación JEL: F37, F47.

Palabras clave: crisis financieras, mercados emergentes, modelos de predicción, modelos de alerta temprana.

* Economista y magíster en finanzas por la Universidad del Pacífico. Profesor auxiliar de la Facultad de Ciencias Económicas. 


\title{
Pensamiento Crítico Vol. I9. No I
}

\begin{abstract}
In this paper we describe one of the most important models on financial crisis prediction in emerging markets: signal approach model; we also present the results of the test of this model using monthly data from 1970 until first quarter of 2009. The suggested model has been a re-estimation of Kaminsky, Lizondo and Reinhart's approach (1998). We obtain an identification of the main predictors of financial crisis (in the empiric sense of the present work) understood as an approximation to the probability of crisis in the short term.
\end{abstract}

Keywords: Financial crises, Emerging markets, Prediction models, Early warning systems.

\section{Classification Number JEL: F37, F47}

\section{Introducción}

La presencia de crisis financieras globales sigue siendo un tema de importancia en la economía mundial, desde bancos centrales de países desarrollados y en desarrollo, bancos de inversión, hasta numerosos centros de investigación y autoridades económicas de varios países monitorean permanentemente el desarrollo de los mercados financieros y emiten análisis y predicciones sobre este fenómeno. Si bien la economía mundial ha pasado por uno de sus períodos más prolongados de crecimiento entre los años 20032008, tal como señala la experiencia histórica, estos períodos también generan condiciones para el surgimiento de crisis financieras a través de la expansión, que podemos llamar "desordenada," de algunos sectores. La reciente crisis financiera en Estados Unidos ha mostrado, en la principal economía capitalista, la naturaleza recurrente de las crisis. También ha puesto en debate el gran problema que enfrenta la economía normativa en el campo de la regulación financiera, la coordinación de políticas no solo macroeconómicas sino también financieras en un marco de mayor integración financiera mundial.

\section{El modelo de predicción de crisis de Kaminsky, Lizondo y Reinhart (1998)}

Desde el punto de vista de los modelos de predicción de crisis financieras, la estimación de un sistema de alerta temprana consta de cuatro pasos. Estos son ${ }^{1}$ :

1 Ver Edison (2000). 


\section{Alfonso Leonel Ayala Loro}

1. La formulación de un sistema de identificación de los acontecimientos considerados como crisis.

2. La elección de un conjunto de variables que se consideran relevantes para anticipar las crisis. Estas son variables macroeconómicas monetarias, financieras, del sector real y externas.

3. La definición de un método estadístico o econométrico que permita calcular la probabilidad de ocurrencia de una crisis en un período determinado.

4. Un procedimiento para evaluar la bondad de las predicciones realizadas.

En el presente trabajo seguimos el modelo de alerta temprana básico de Kaminsky, Lizondo y Reinhart (1998), quienes desarrollan un modelo basado en un trabajo anterior de Kaminsky y Reinhart (1996), usando el concepto de "umbrales" para generar un marco de trabajo en el que se evalúa un conjunto de indicadores "candidatos" a predictores de ocurrencia de una crisis.

Una crisis es definida como un período de extrema presión en el mercado cambiario (exchange market pressure). El índice que reproduce el estado de crisis es calculado como el promedio ponderado de cambios porcentuales mensuales en las reservas internacionales y el cambio porcentual en el tipo de cambio, relacionando los ponderadores tal que los dos componentes del índice tengan la misma volatilidad. El índice de presión del mercado cambiario (Exchange market pressure, EMP) es:

$$
e m p=\Delta \% e_{t}+\alpha_{1} \Delta \% r_{t}
$$

donde $\alpha_{1}$ es el ratio de las desviaciones muestrales de la tasa de cambio mensual del tipo de cambio y las reservas internacionales. Cuando el índice EMP sobrepasa un umbral máximo definido como el valor de la media de EMP más un número de veces la desviación estándar de este indicador, se define que se ha iniciado una crisis $\mathrm{si}^{2}$ :

2 En el tratamiento empírico puede suceder que la intensidad de una crisis haga que el indicador sobrepase su umbral durante varios periodos (meses) después de iniciada una crisis, por lo que será necesario definir un periodo mínimo de separación entre una crisis y la siguiente, otros modelos incluyen esta información 


\section{Pensamiento Crítico Vol. 19. N I}

$$
\text { Crisis }=\left\{\begin{array}{l}
1 \text { si emp } \mathrm{em}_{t}>\mu_{\text {emp }}+\mathrm{n} \text { veces } \mathrm{o}_{e m p} \\
0 \text { en otro caso }
\end{array}\right.
$$

se da una señal por emitida cuando el índice sobrepasa el umbral, es decir:

$$
S_{t}=\left\{\begin{array}{l}
1 \text { si }\left|X_{t}\right|>|\bar{X}| \\
0 \text { si }\left|X_{t}\right| \leq|\bar{X}|
\end{array}\right.
$$

Donde $\bar{X}$ es a la vez un umbral definido por el valor que asume el n-ésimo percentil de la distribución del indicador en estudio, donde el percentil es común a todos los países, pero el valor del indicador es diferente para cada país, ya que corresponde a la distribución particular de la variable en estudio.

Según este enfoque se puede evaluar algunas de las propiedades deseables para las señales de cada indicador potencial, como por ejemplo la persistencia de una señal. Idealmente una señal debe ser emitida en el período "ventana" elegido y acertar exactamente al cumplimiento del mismo. Por ejemplo, si varios indicadores emiten una señal en junio de 1991, la crisis debe producirse en julio de 1993 y la persistencia de la señal emitida debe durar el mismo tiempo que dura la crisis, es decir debe ser perfectamente predecible el momento y la duración de cada crisis, asimismo no debe dejar de explicar todas las crisis que cumplen su definición empírica.

Para analizar la efectividad de las señales emitidas por un indicador potencial, tenemos que considerar que una variable puede "emitir" una señal (un 0 o un 1 , una variable binaria) cuando ha pasado un umbral dado (sobre o por debajo del umbral). La tabla siguiente resume los posibles resultados:

\section{TABLA 1. MATRIZ DE EVALUACIÓN DE SEÑALES DEL MODELO DE KAMINSKY, LIZONDO Y REINHART (1998)}

\begin{tabular}{|c|c|c|}
\hline & $\begin{array}{c}\text { Ocurrió una crisis en los siguientes 24 } \\
\text { meses. }\end{array}$ & $\begin{array}{c}\text { No ocurrió una crisis en los siguientes } \\
24 \text { meses. }\end{array}$ \\
\hline $\begin{array}{c}\text { La señal fue emitida en los 24 } \\
\text { meses anteriores a la crisis }\end{array}$ & A & $\begin{array}{c}\text { B } \\
\text { (Error tipo I) }\end{array}$ \\
\hline $\begin{array}{c}\text { La señal no fue emitida en los } 24 \\
\text { meses anteriores a la crisis }\end{array}$ & $\begin{array}{c}\text { C } \\
\text { (Error tipo II) }\end{array}$ & D \\
\hline
\end{tabular}

como indicativo de la intensidad de la crisis. 


\section{Alfonso Leonel Ayala Loro}

Donde A es el número de meses que el indicador emitió una buena señal dado que se produce una crisis en la ventana de predicción establecida, en este caso 24 meses. B es el número de meses que el indicador emitió una mala señal o "ruido," es decir no se produjo una crisis. $\mathrm{C}$ es el número de meses en los cuales el indicador no emitió una señal que hubiera sido una verdadera. $\mathrm{D}$ es el número de meses que el indicador no emitió una señal que hubiera sido mala. Para cada indicador KLR encuentran un umbral "óptimo", definido como el umbral que minimiza el coeficiente ruido-señal $B /(B+D) /$ $\mathrm{A} /(\mathrm{A}+\mathrm{C})$, a base de hacer una búsqueda grid o malla entre los percentiles más bajos o altos de la distribución, según lo que indique el marco teórico desarrollado.

Cuando tenemos que elegir el valor óptimo del percentil para cada variable, surge el problema de incurrir en los errores de tipo I y II, sea a el error tipo I y b el error tipo II, entonces $\mathrm{a}^{\prime}(\bar{X})>0$, y $\mathrm{b}^{\prime}(\bar{X})<0$. Podemos ponderar las señales por el ratio $\mathrm{w}=\mathrm{b} /(1-$ a) tratando de minimizarlo, que es el procedimiento operativo que da forma al modelo.

En la tabla anterior la probabilidad de incurrir en un error de tipo I es $\mathrm{P}$ (Rechazar Ho / Ho es verdadera), en tanto que el error de tipo II es $\mathrm{P}$ (No rechazar Ho / Ho es falsa), el primer elemento es $\mathrm{C} /(\mathrm{A}+\mathrm{C})$ y el segundo $\mathrm{B} /(\mathrm{B}+\mathrm{D})$, queremos minimizar el ratio ruido (Error tipo II) a señal (1 - Error tipo I).

Dentro de un intervalo determinado para el percentil ${ }^{3}$, el criterio para elegir el percentil específico viene dado por el objetivo de minimizar el denominado ratio de "ruido/señal" que viene dado por la siguiente expresión:

$$
\text { Min } \frac{\text { Error tipo II }}{1-\text { Error Tipo I }}=\frac{\frac{B}{B+D}}{1-\left(\frac{C}{A+C}\right)}=\frac{\frac{B}{B+D}}{\left(\frac{A}{A+C}\right)}
$$

3 En línea con los estudios realizados en este tipo de modelación, la búsqueda grid se realizará entre los percentiles décimo y vigésimo en el caso de los percentiles inferiores. 


\section{Pensamiento Crítico Vol. 19. N I}

Un indicador sería perfecto sí únicamente "emitiera" señales (1s) que se ubicaran en las celdas A y D de la tabla, también podría suceder que un indicador emita en todos los meses incluidos en la "ventana", tal que A es mayor a cero y C igual a cero, también podría no emitir señales dado que no hay crisis en los siguientes meses, esto es, $B=0$ y $\mathrm{D}>0$. No obstante, es imposible que algún indicador tenga un comportamiento ideal.

Otro indicador de performance de un indicador potencial es el número de buenas señales emitidas como porcentaje del número total de observaciones que pudieron ser emitidas $\mathrm{A} /(\mathrm{A}+\mathrm{C})$, por último, otro indicador de nuestro interés es la probabilidad condicional que una crisis pueda ser identificada $A /(A+B)$.

Es de interés calcular la probabilidad condicional de que se produzca una crisis en el horizonte de predicción. Para ello el conjunto de indicadores potenciales que teóricamente emiten señales necesitan ponderarse en función de su desempeño como buenos indicadores de crisis. Cada señal emitida es ponderada entonces por el ratio ruido-señal, tal que los indicadores con mayor ratio son valorados con una menor probabilidad agregada.

Como se ha señalado, el coeficiente ruido-señal es una medida de la capacidad de un indicador en emitir buenas señales y evitar las malas señales, el porcentaje de señales falsas sobre el porcentaje de buenas señales, en términos de los elementos de la tabla 1 es, $[(\mathrm{B} / \mathrm{B}+\mathrm{D}) /(\mathrm{A} / \mathrm{A}+\mathrm{C})]$. Aquellos indicadores con un coeficiente ruido-señal mayor de la unidad emiten demasiadas señales falsas, por lo que no son confiables para predecir una crisis.

Asimismo se debe comparar la probabilidad condicional con la probabilidad incondicional de una crisis ${ }^{4}$. Para que un indicador sea considerado útil para predecir una crisis, la probabilidad condicional debe ser mayor que la probabilidad incondicional, esto es, $A / A+B>(A+C) /(A+B+C+D)$. Si el indicador no es sensible a emitir señales falsas, lo que implica que habrá pocas señales calificadas como " $\mathrm{B}$ " (el indicador emite una señal cuando no hay crisis), la probabilidad condicional será cercana a 1.

4 La probabilidad incondicional está dada por la proporción (simple) de periodos de crisis en el lapso del estudio. 


\section{Alfonso Leonel Ayala Loro}

De este modo, si el indicador es fiable la probabilidad condicional será mayor que la probabilidad incondicional.

Por otro lado, al tratarse de un modelo no paramétrico, no es posible calcular un estadístico de ajuste global del modelo del tipo $\mathrm{R}^{2}$ o similares, por lo que el análisis de los resultados debe considerar los dos aspectos antes mencionados. Sin embargo, el trabajo de Berg, Borensztein y Pattillo (2004) implementa un método alternativo especial para evaluar una "bondad de ajuste" mediante el cálculo de una "función de pérdida" y un test estadístico a la probabilidad estimada de crisis del modelo mediante un modelo data panel simple.

En el presente documento tomamos una muestra de 20 países emergentes, estos son los siguientes: Argentina, Bolivia, Brasil, Colombia, Chile, India, Indonesia, Israel, Jordania, Corea, Malasia, México, Perú, Filipinas, Singapur, Sudáfrica, Tailandia, Turquía, Uruguay y Venezuela. Un primer criterio para la elección de los países fue el que hayan pasado por al menos una crisis financiera en el período de estudio. Esto si bien puede agregar un sesgo de especificación, puede verse minimizado con la diversidad de países que se considere. Un segundo criterio es que representen economía significativas en sus respectivas áreas geográficas, de esta manera se excluyen pequeños Estados con poca participación en la economía mundial. Las economías que se analizan tienen una participación creciente en la economía mundial, llegando a alcanzar un $20.56 \%$ al año 2009, desde un $16.52 \%$ en 1980 , según el método de paridad de poder de compra utilizado en el Fondo Monetario Internacional. Esta mayor participación corresponde a la creciente importancia de las economías del sudeste asiático y la India, en contraposición con la pérdida de participación que han experimentado los países de América Latina.

El gráfico 1 muestra la evolución de cada economía desde 1980, según datos del World Economic Outlook de Octubre del $2009^{5}$.

5 Disponible en: http://www.imf.org/external/pubs/ft/weo/2009/02/weodata/index.aspx 


\section{Pensamiento Crítico Vol. 19. No I}

\section{GRÁFICO 1. PARTICIPACIÓN EN EL PBI MUNDIAL DE LAS 20 ECONOMÍAS EMERGENTES DE LA MUESTRA: 1980-2009}

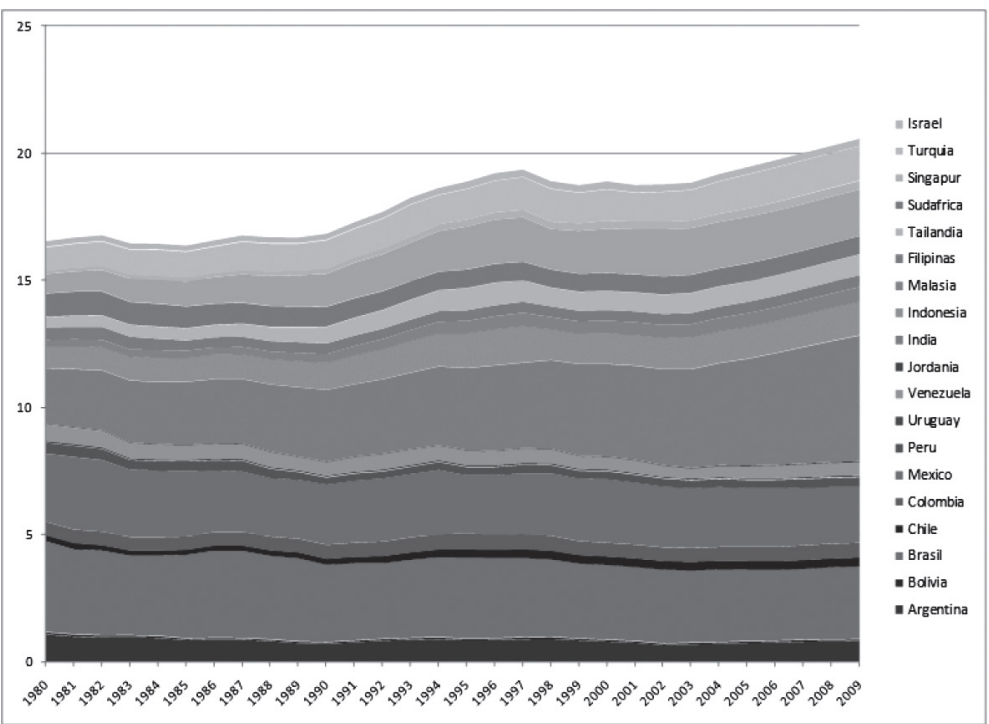

\section{La revisión de la modelación de sistemas de alerta temprana de Berg, Borensztein y Pattillo (2004)}

En un trabajo de revisión de los modelos de alerta temprana producidos hasta el año 2004, Berg, Borenzstein y Pattillo comparan la diversidad de modelos disponibles para producir sistemas de alerta temprana con la intención de revelar algunas ventajas o desventajas de su uso por parte de los organismos internacionales, en especial el FMI, quien también efectúa un seguimiento a los pronósticos de modelos del sector privado (el Emerging Market Risk Indicator del Credit Suisse First Boston, el GS-WATCH del Banco de Inversión Goldman Sachs y el Alarm Clock del Deutsche Bank).

Después de analizar la performance de los modelos "out-of-sample" para las predicciones entre enero de 1999 y diciembre del 2000 para un modelo KLR y DCSD", los autores señalan que los resultados "son mixtos", las predicciones del modelo KLR

6 Developing Country Studies Division que es la división del Fondo Monetario que efectúa el cálculo del modelo de predicción de crisis del Fondo. 


\section{Alfonso Leonel Ayala Loro}

son estadísticamente y económicamente significativas de las crisis actuales. Sin embargo, la precisión en el período "out-of-sample" es solo ligeramente inferior al ajuste en el período de estimación, en tanto que el modelo de la DCSD se desempeña de manera muy inferior en el periodo out-of-sample. Bajo los resultados mostrados, las predicciones son aun significativas, sin embargo la hipótesis de que las predicciones son insesgadas $e$ informativas tiene una probabilidad mayor que la hipótesis que las predicciones son inútiles. Asimismo la evaluación respecto a los modelos privados de corto plazo muestra una performance pobre.

\section{La crítica de Pistelli (2006)}

Pistelli (2006) apunta que los modelos desarrollados bajo la influencia del enfoque de señales de KLR y otros tienen pocos fundamentos teóricos y problemas de aplicación, cuestionando 3 puntos principales: (a) el uso de variables en variaciones a 12 meses, ya que según el autor no hay fundamentos teóricos para afirmar que la variación en un año es una variable adecuada en este caso, debiendo ser el indicador formulado en términos de niveles; (b) problemas con la medición de sobrevaluación del tipo de cambio real, señalando que al tomar el período completo de análisis para tomar las variaciones respecto a una tendencia determinística se incluirá el "salto" futuro del tipo de cambio real, produciendo por construcción buenos resultados, y (c) el problema del sesgo postcrisis, este se produce ya que el modelo toma, como hemos visto dos tipos de períodos "tranquilos" e "intranquilos", incluyendo en este ultimo los períodos inmediatamente posteriores al inicio de las crisis, según muestra el autor, este hecho produce un sesgo en los resultados ya que "esto puede llevar a un importante sesgo

porque el comportamiento de las variables independientes es muy diferente durante los períodos tranquilos en comparación con los períodos de recuperación"7.

Para solucionar estos inconvenientes en el modelo de KLR, Pistelli propone analizar otros indicadores de crisis, clasificados en cuatro grupos: a) adecuación de las reservas internacionales, b) sector externo y competitividad internacional, c) sector financiero doméstico y d) sector real doméstico.

El autor estima una medida de performance basada en el cálculo de las diferencias entre el valor promedio en períodos de crisis y períodos tranquilos estandarizados,

$7 \quad$ Ibid, pag 10, traducción del autor. 


\section{Pensamiento Crítico Vol. 19. N I}

mostrando que los indicadores propuestos se comportan mejor que los indicadores incluidos en el trabajo de Kaminsky y Reinhart (1999), luego de ello ordena la eficiencia de los indicadores basado en un promedio del ratio ruido/señal como la diferencia estandarizada, para indicar que solo el ratio de la desviación del tipo de cambio real ${ }^{8}$, se encuentra entre los mejores seis indicadores de crisis.

El autor concluye, luego de comparar el indicador multivariado que toma ambos enfoques con los seis mejores indicadores de Kaminsky y Reinhart (denominados KR-6), que el primero se ajusta mejor a la predicción de crisis tanto en la precisión de la señal, como en la precedencia durante la crisis, ya que llega a emitir por lo menos una señal en todas las crisis identificadas, así como al menos tres señales en al menos $68 \%$ de las crisis, contra solo un $47 \%$ de los casos para el modelo "KR-6".

\section{Resultados del modelo}

Usando datos mensuales para los 20 países de una muestra de mercados emergentes $^{9}$, se calculó el índice de presión en el mercado cambiario, o como se denomina en la literatura el índice EMP, siguiendo a Kaminsky, Lizondo y Reinhart (1996) y Edison (2000), estimamos un umbral determinando un múltiplo de las desviaciones estándar de la ecuación (2)tal que pueda captar los últimos episodios de crisis ${ }^{10}$, el múltiplo seleccionado en el presente trabajo es de 2.20, en el gráfico 2 se muestra los resultados para los países de la muestra, así como los umbrales máximos tanto para los períodos de inflación baja, como para los períodos de alta inflación, lo que determina que algunos países muestren dos umbrales. Se identificaron 124 episodios de crisis en el período analizado, aproximadamente 30 episodios más que el estudio de Edison del año 2000. La tabla 2 a continuación muestra los períodos identificados y el año y mes de su inicio para cada país.

8 Calculado como la diferencia entre el tipo de cambio real y el promedio móvil de los últimos 48 meses y no como lo calcula Kaminsky y Reinhart (1999) respecto a una tendencia determinística tomando toda la información existente.

9 Argentina, Brasil, Bolivia, Chile, Colombia, India, Indonesia, Israel, Jordania, Corea, Malasia, México, Perú, Filipinas, Sudáfrica, Singapur, Tailandia, Turquía, Uruguay y Venezuela.

10 Tal como reporta Edison, op. cit., es necesario revisar en cada estimación a realizar, el valor del mencionado múltiplo. Al agregarse mayor información, si ésta corresponde a periodos sin crisis financieras, es de esperarse que el múltiplo deba caer, al ajustar a este nuevo valor se evita introducir "observaciones" de periodos de crisis falsas en la estimación. 
GRÁFICO 2. IDENTIFICACIÓN DE CRISIS POR PAÍSES 1970-2009

Indice EMP: Argentina 1970-2009

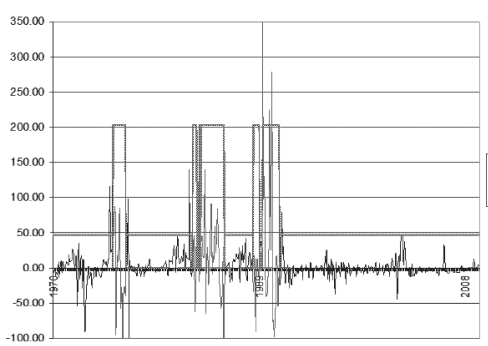

Indice EMP: Brasil 1970-2009

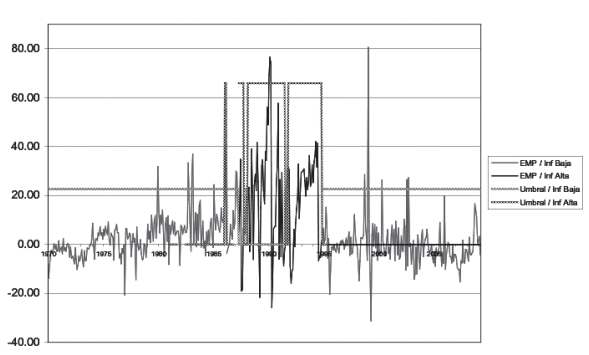

Indice EMP: Colombia 1970-2009

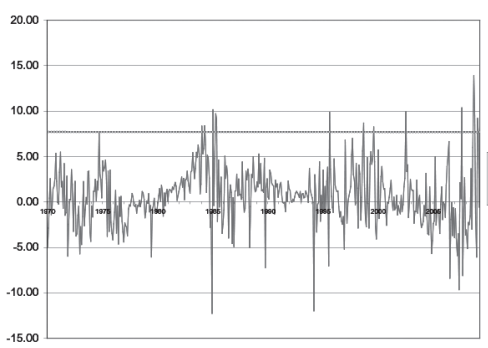

Indice EMP: Bolivia 1970-2009

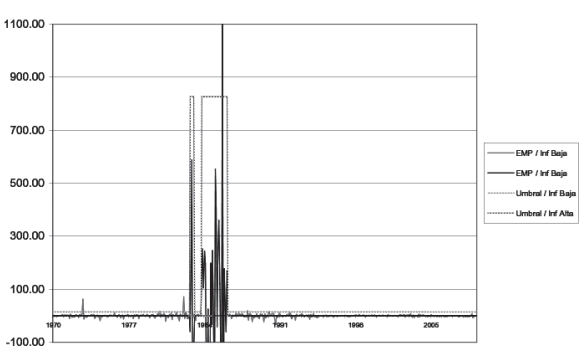

Indice EMP: Chile 1970-2009

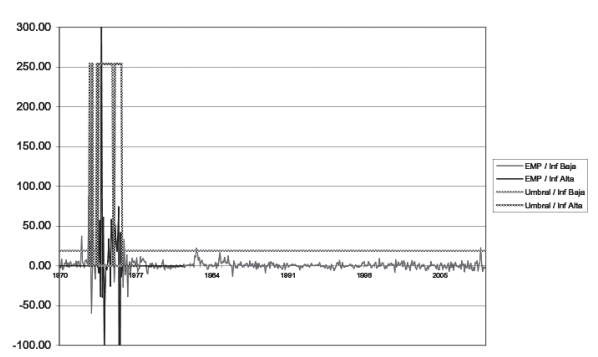

Indice EMP: India 1970-2009

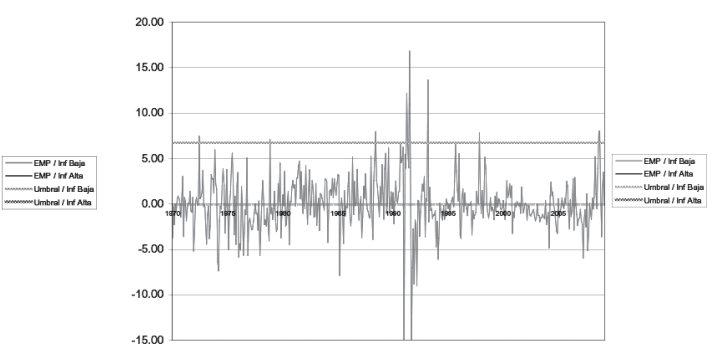




\section{Pensamiento Crítico Vol. 19. $\mathrm{N}^{\circ}$ I}
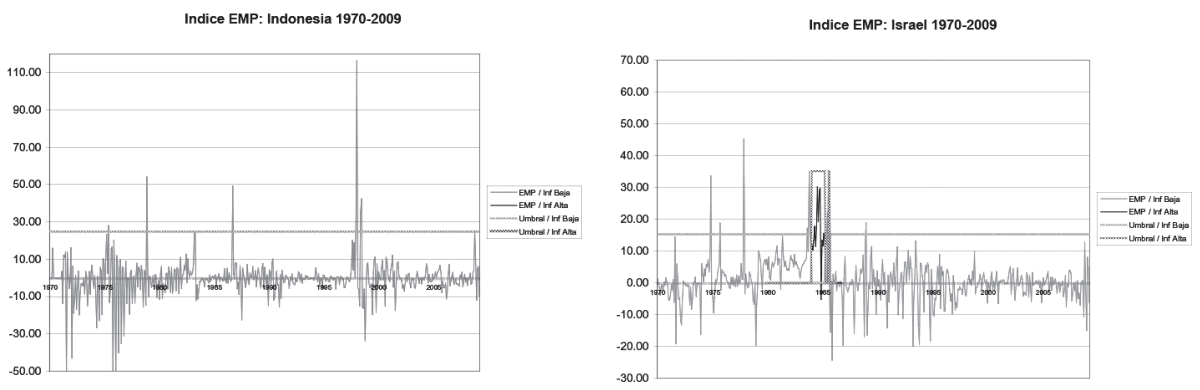

Indice EMP: Jordania 1970-2009
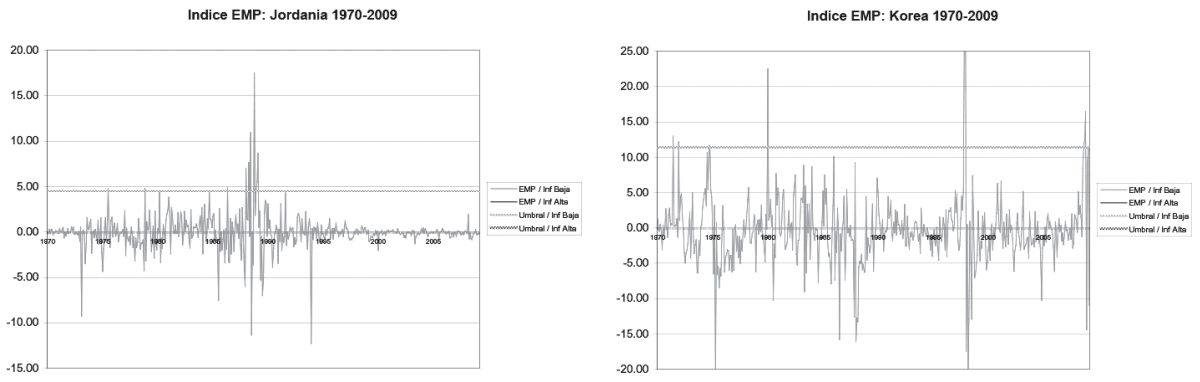

Indice EMP: Malasia 1970-2009
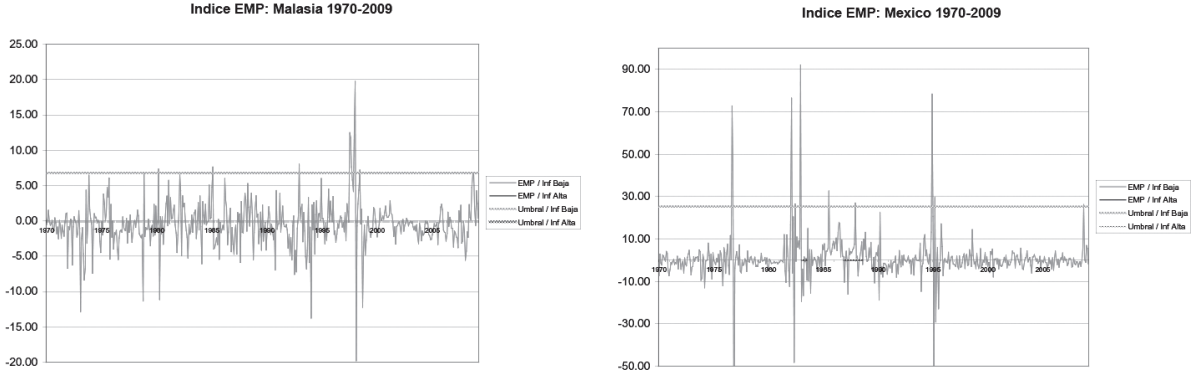


\section{Alfonso Leonel Ayala Loro}

Indice EMP: Perú 1970-2009

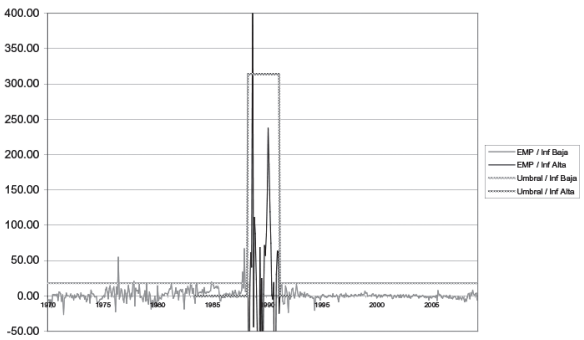

Indice EMP: Sudafrica 1970-2009

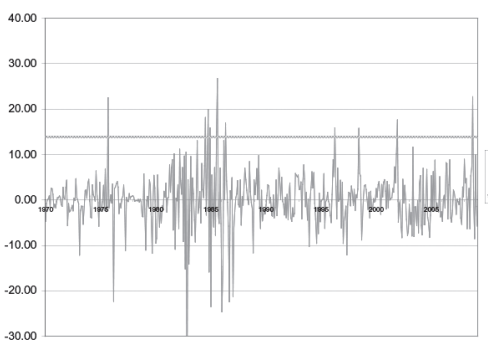

Indice EMP: Filipinas 1970-2009

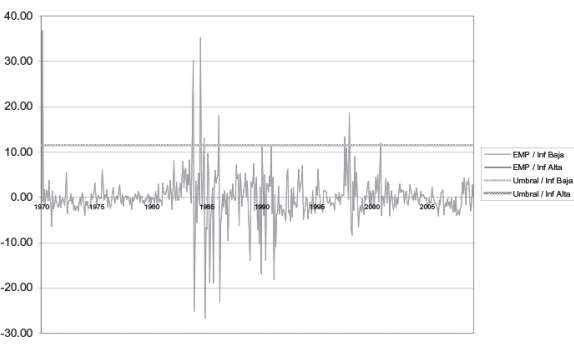

Indice EMP: Singapur 1970-2009

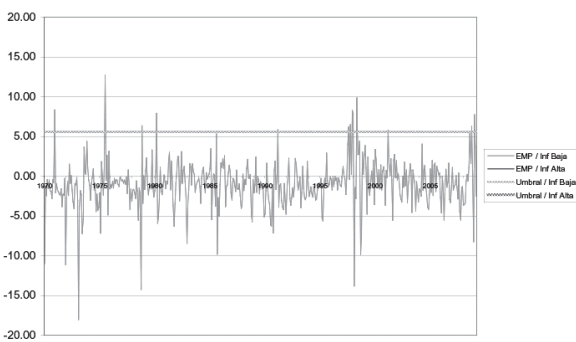

Indice EMP. Tailandia 1970-2009

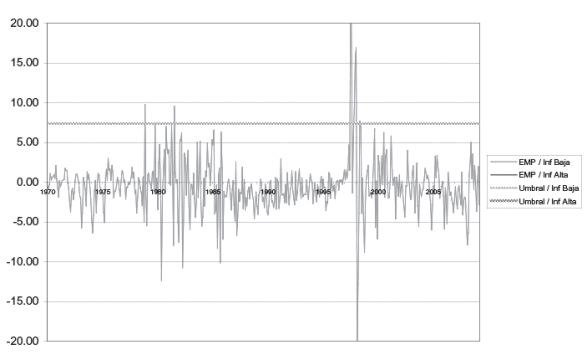

Indice EMP: Turquía 1970-2009

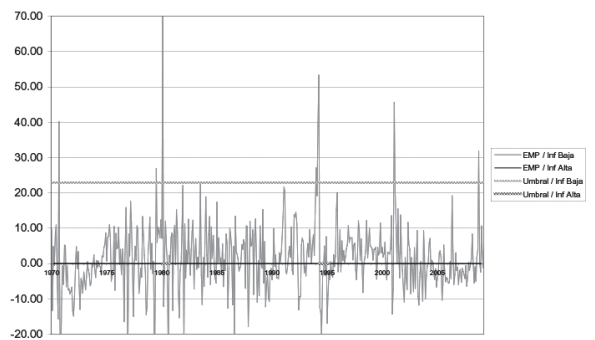



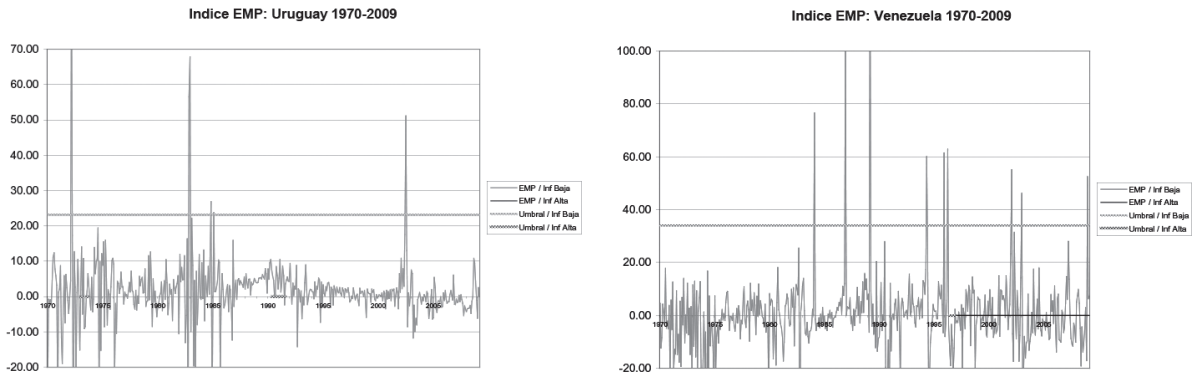

Sobre el modelo propuesto cabe comentar que en general identifica adecuadamente las fechas de inicio de crisis en la mayoría de países, pero en algunos casos no puede identificar algunos episodios aislados de alta presión en el mercado cambiario como crisis, así por ejemplo en el caso de Israel a finales del 2008, o la presión cambiaria de Argentina del 2006, lo que algunos medios de comunicación en esos años clasificaban como crisis.

TABLA 2. FECHAS DE INICIO DE CRISIS IDENTIFICADOS EN EL MODELO POR PAÍSES, 1970 - 2009

\begin{tabular}{|c|c|c|c|c|c|}
\hline País & Argentina & Bolivia & Brasil & Chile & Colombia \\
\hline Fecha & $\begin{array}{r}\text { mar-75 } \\
\text { nov-76 } \\
\text { jul-82 } \\
\text { abr-89 } \\
\text { dic-89 } \\
\text { ene-91 } \\
\text { feb-02 }\end{array}$ & $\begin{array}{l}\text { oct-72 } \\
\text { nov-79 } \\
\text { feb-82 } \\
\text { sep-85 } \\
\text { ene-88 }\end{array}$ & $\begin{array}{c}\text { dic-79 } \\
\text { sep-82 } \\
\text { ene-85 } \\
\text { ene-87 } \\
\text { ene-88 } \\
\text { ene-90 } \\
\text { sep-91 } \\
\text { sep-98 } \\
\text { abr-00 } \\
\text { jul-02 }\end{array}$ & $\begin{array}{c}\text { ene-72 } \\
\text { sep-72 } \\
\text { jul-73 } \\
\text { dic-74 } \\
\text { oct-75 } \\
\text { ago-82 } \\
\text { oct-08 }\end{array}$ & $\begin{array}{c}\text { ene-84 } \\
\text { ene-85 } \\
\text { ago-95 } \\
\text { sep-98 } \\
\text { ago-99 } \\
\text { jul-02 } \\
\text { ago-07 } \\
\text { sep-08 }\end{array}$ \\
\hline$N^{0}$ de crisis & 7 & 5 & 10 & 7 & 8 \\
\hline
\end{tabular}




\begin{tabular}{|l|lcc|c|c|}
\hline País & India & Indonesia & Israel & Jordania & Korea \\
\hline Fecha & & & & & \\
& jun-72 & may-75 & nov-74 & jul-75 & sep-74 \\
& nov-78 & nov-78 & sep-75 & nov-78 & ene-80 \\
& jun-88 & sep-86 & nov-77 & mar-80 & nov-97 \\
& sep-90 & dic-97 & ago-83 & may-86 & sep-08 \\
& abr-91 & oct-08 & jun-85 & ene-88 & \\
& mar-93 & & dic-88 & & \\
& nov-97 & & & & \\
& sep-08 & & & & \\
& & & & & \\
& & & & & \\
& & 5 & 6 & 5 & 4 \\
\hline
\end{tabular}

\begin{tabular}{|c|c|c|c|c|c|}
\hline País & Malasia & Mexico & Perú & Filipinas & Sudafrica \\
\hline Fecha & $\begin{array}{c}\text { mar-80 } \\
\text { feb-85 } \\
\text { dic-92 } \\
\text { jul-97 } \\
\text { oct-08 }\end{array}$ & $\begin{array}{l}\text { sep-76 } \\
\text { feb-82 } \\
\text { dic-82 } \\
\text { jul-85 } \\
\text { dic-87 } \\
\text { dic-94 } \\
\text { oct-08 }\end{array}$ & $\begin{array}{l}\text { jun-76 } \\
\text { nov-77 } \\
\text { dic-84 } \\
\text { oct-87 } \\
\text { sep-88 }\end{array}$ & $\begin{array}{c}\text { sep-83 } \\
\text { jun-84 } \\
\text { feb-86 } \\
\text { jul-97 } \\
\text { oct-00 }\end{array}$ & $\begin{array}{c}\text { sep-75 } \\
\text { jul-84 } \\
\text { jul-85 } \\
\text { may-86 } \\
\text { abr-96 } \\
\text { jun-98 } \\
\text { dic-01 } \\
\text { oct-08 }\end{array}$ \\
\hline $\mathrm{N}^{0}$ de crisis & 5 & 7 & 5 & 5 & 8 \\
\hline
\end{tabular}




\begin{tabular}{|l|ccccc|}
\hline País & Singapur & Tailandia & Turquia & Uruguay & Venezuela \\
\hline Fecha & & & & & \\
& jul-75 & nov-78 & jul-79 & mar-72 & feb-84 \\
& nov-78 & oct-79 & feb-80 & nov-82 & dic-86 \\
& mar-80 & jul-81 & ene-94 & nov-84 & mar-89 \\
& mar-91 & jul-97 & feb-01 & jul-02 & may-94 \\
& ago-97 & & oct-08 & & dic-95 \\
& mar-01 & & & & feb-02 \\
& oct-08 & & & & ene-03 \\
& & & & & ene-09 \\
& & & & & \\
& & & & & \\
& & & & & \\
\end{tabular}

El proceso de análisis de cada indicador se basó primeramente en la clasificación de sus tasas de crecimiento o desviaciones (excepto en el caso de la tasa de interés que se toma la información a en niveles) como predictores posibles de crisis. La tabla 3 muestra para cada uno de los 15 indicadores propuestos la región de interés de la distribución empírica, tanto de las tasas de crecimiento como de las desviaciones, dependiendo de cada caso.

TABLA 3. INDICADORES PROPUESTOS Y REGIÓN DE INTERÉS COMO PREDICTORES DE CRISIS

\begin{tabular}{|c|l|c|}
\hline \multicolumn{2}{|c|}{ Indicador } & $\begin{array}{c}\text { Región de interés como predictor de } \\
\text { crisis }\end{array}$ \\
\hline 1 & Tasa de interés en Estados Unidos & Superior \\
\hline 2 & Tasas de interés domésticas & Superior \\
\hline 3 & Crédito doméstico / PBI & Superior \\
\hline 4 & Desviación del tipo de cambio real & Inferior \\
\hline 5 & Diferencial de tasas de interés domesticas & Superior \\
\hline 6 & Multiplicador de M2 & Superior \\
\hline
\end{tabular}




\section{Alfonso Leonel Ayala Loro}

\begin{tabular}{|c|l|c|}
\hline 7 & Precio del petróleo & Superior \\
\hline 8 & Importaciones & Superior \\
\hline 9 & Ratio tasas activas sobre pasivas & Superior \\
\hline 10 & Reservas internacionales & Inferior \\
\hline 11 & Depósitos bancarios & Inferior \\
\hline 12 & Ratio M2 sobre reservas internacionales & Superior \\
\hline 13 & Exportaciones & Inferior \\
\hline 14 & Producción industrial & Inferior \\
\hline 15 & Índice bursátil & Inferior \\
\hline
\end{tabular}

Para las crisis identificadas se procedió a calcular el número de ocurrencias en las que el valor del indicador sobrepasaba o caía por debajo de un umbral determinado, realizando una simulación grid tanto en el segmento superior de los percentiles como en el inferior, dependiendo de la naturaleza del indicador. Esto corresponde a la estimación del valor de las celdas A y $\mathrm{C}$ de la tabla 1 . En el caso de que las crisis tengan períodos superpuestos, se procedió a separar cada crisis y tratar a cada una como un evento singular.

Según la metodología del enfoque de señales, se debe evaluar los resultados de cada indicador a través de varios parámetros; primero, el ratio ruido-señal, y en segundo lugar a través del porcentaje de crisis en la cual el indicador emitió al menos una señal de crisis, asimismo por el porcentaje de crisis detectadas, entre otros aspectos. Los resultados de nuestro modelo se muestran en la tabla 4. 
Pensamiento Crítico Vol. 19. N I

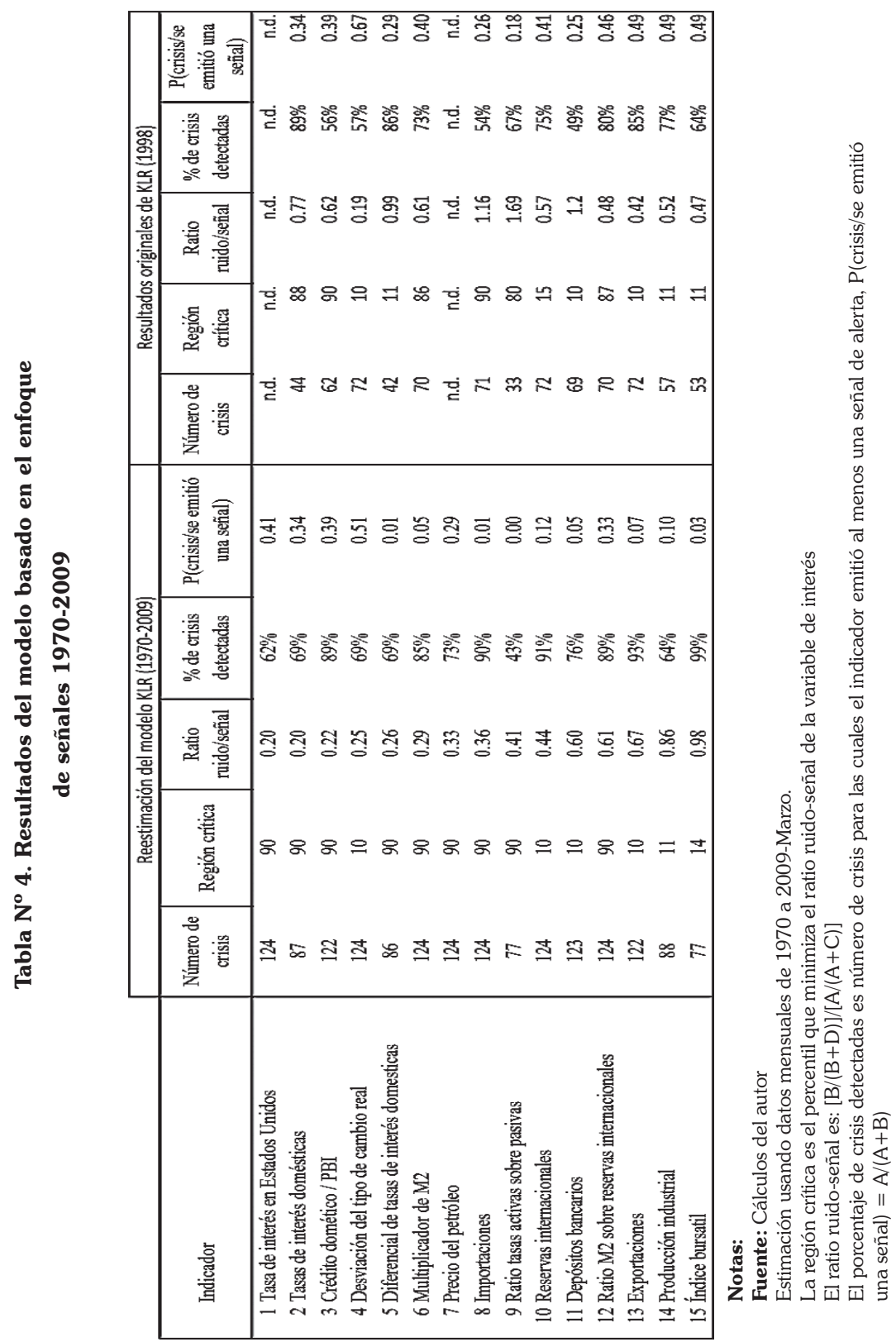




\section{Alfonso Leonel Ayala Loro}

Para el período en estudio, la tabla muestra en la primera columna el número de crisis para los que existe información disponible. Así, por ejemplo, el indicador para el cual existe menor información es el índice bursátil con información disponible solo para 77 crisis, en tanto que la mayoría de indicadores está disponible para la totalidad de crisis identificadas. En la segunda columna se puede observar la región crítica, en términos del percentil de la distribución de frecuencia de la variable de interés. Por ejemplo, para el caso de la tasa de interés de Estados Unidos el percentil que define la región crítica es el 90, en tanto que el percentil para el indicador de la tasa de crecimiento es el 10, esto es la cola inferior de la distribución. En la tercera columna se muestra el ratio ruido-señal para el percentil obtenido, cabe resaltar que la búsqueda se realizó entre el percentil 10 y 20 para el caso de percentiles bajos y entre 80 y 90 para el caso de los percentiles altos. Como vemos en la tercera columna, el ratio ruido-señal varía ampliamente entre las variables estudiadas, siendo el ratio de mejor performance el de la tasa de interés de Estados Unidos, seguida de cerca de la tasa de interés doméstica y el ratio de crédito doméstico/PBI, la mayoría de los indicadores muestra un ratio ruido-señal bastante bajo respecto a estudios anteriores (esto se puede deber a la mayor disponibilidad de datos). Asimismo, todos los indicadores propuestos muestran un ratio menor de uno, por lo que no puede descartarse, según este indicador de ajuste, la inclusión en el modelo de la información proporcionada por estos. Otro aspecto a observar es el porcentaje de crisis para el cual se emite al menos una señal de alarma. Este valor también varía ampliamente entre las variables elegidas, así el indicador que muestra un menor porcentaje es el ratio de tasas activas sobre pasiva del país, con solo $42 \%$ : en tanto la tasa de interés de Estados Unidos solo detecta el $62 \%$ de las crisis y la producción industrial solo un $64 \%$. Finalmente, la última columna muestra la probabilidad de que se dé una crisis dado que se emite una señal de alerta, descontando la probabilidad no condicional de que se produzca una crisis, con los datos disponibles esta probabilidad es de $27.91 \%$.

Varios comentarios se pueden ofrecer a la luz de estos resultados; por ejemplo, en general, los valores de los percentiles estimados son muy parecidos a los resultados de KLR (1996), Berg y Pattillo (1998) y Edison (2000), pero una diferencia importante es el valor de los ratios ruido-señal, estos han mejorado considerablemente con respecto a los trabajos anteriores, sin embargo esto no se traduce en una mejora del porcentaje de crisis detectadas para la última crisis financiera global del 2008. Esto es un indicativo de que el poder predictivo del modelo puede no ser lo suficientemente alto para detectar nuevas crisis, tema al que llegaremos en el siguiente acápite. Asimismo el orden relativo 


\section{Pensamiento Crítico Vol. 19. N I}

según el ajuste del ratio ruido-señal varía respecto a los trabajos anteriores. El indicador de las tasas de interés de referencia en Estados Unidos es el que muestra un mejor nivel de ajuste relativo (variable no considerada en el trabajo original de KLR e incorporada en el estudio de Edison); también las tasas de interés domésticas muestran un buen nivel de ajuste, aunque la disponibilidad de data es una de las más bajas en el estudio. El ratio de crédito doméstico sobre PBI mantiene su importancia relativa entre ambos estudios. El indicador para la desviación del tipo de cambio cae al cuarto lugar, mientras en los estudios anteriores era la primera o segunda variable de importancia, también puede notarse que las variables financieras ocupan la mayoría de los primeros lugares en cuanto a la efectividad para predecir las crisis, entre las variables de predicción estudiadas.

Cabe comentar que el poder predictivo de la variable ratio de tasas de interés activas sobre pasivas es nulo, ya que la probabilidad no condicional es superior a la probabilidad condicional de que se produzca una crisis, si bien el ratio ruido-señal es menor de uno, la significancia estadística general de la variable es cero, por lo que se puede descartar su inclusión en el modelo.

\section{Conclusiones}

Las variables macroeconómicas globales, tales como la tasa de interés de referencia de Estados Unidos y variables domésticas como el crédito interno medido como porcentaje del PBI, la desviación del tipo de cambio real y la tasa de interés real doméstica, en un modelo en la tradición del enfoque de señales, muestran una mayor importancia relativa y un buen nivel de ajuste para estimar la probabilidad de crisis financieras en mercados emergentes.

El modelo de predicción de crisis planteado ha mostrado, entonces, resultados mixtos; no obstante su aplicación constituye un elemento valioso, ya que este tipo de modelos permite tener elementos prospectivos de análisis de riesgo de crisis en el corto plazo.

Las variables económicas utilizadas permiten, sin embargo, tener una aproximación al efecto que pueden tener cambios en el estado de los mercados financieros en la probabilidad de crisis financieras, aunque la aplicación se ha visto limitada por la disponibilidad de datos. 


\section{Alfonso Leonel Ayala Loro}

La aplicación futura de este tipo de modelación se puede potenciar por la disponibilidad de nuevas series de tiempo, principalmente variables financieras, como variables de la dinámica política de cada país, pueden contribuir en la aplicación de esta metodología en otros mercados emergentes.

\section{Referencias bibliográficas}

BERG, Andrew, BORENSZTEIN, Eduardo; PATTILLO, Catherine (2004) Assessing Early Warning Systems: How Have They Worked in Practice?, IMF Working Paper $\mathrm{WP} / 04 / 52$.

BERG, Andrew; PATTILLO, Catherine (1998) Are currency crises predictable? A test, IMF Working Paper WP/98/154.

EDISON Hali J. (2000) Do Indicators of Financial Crises Work? An Evaluation of an Early Warning System, International Finance Discussion Paper No. 675, Board of Governors of the Federal Reserve System.

FLOOD, Robert P.; MARION, Nancy (1998) Perspectives on the Recent Currency Crises Literature, NBER Working Paper No. 6380, National Bureau of Economic Research.

International Monetary Fund (2009) International Financial Statistics, CD-ROM, Setiembre.

KAMINSKY, Graciela L.; LIZONDO, Saul, REINHART, Carmen (1998) Leading Indicators of Currency Crises, IMF Staff Papers, Vol. 45, pp. 1-48.

PISTELLI, Alfredo (2006) Speculative Currency Attacks: Role of Inconsistent Macroeconomic Policies and Real Exchange Rate Overvaluation, Banco Central de Chile Documento de Trabajo No 379. 